\title{
Acoustic properties of plates with unevenly distributed macroperforations backed by woven meshes
}

\author{
Heidi Ruiz ${ }^{\text {a) }}$ and Pedro Cobo \\ Centro de Acústica Aplicada y Evaluación no Destructiva (CAEND), CSIC-UPM, \\ Serrano 144, 28006 Madrid, Spain \\ Thomas Dupont \\ Département de Recherche en Ingénierie des Véhicules pour l'Environnement, ISAT, Université de Bourgogne, \\ 49 Rue Mademoiselle Bourgeois, 58027 Nevers Cedex, France \\ Bruno Martin \\ Institut Carnot de Bourgogne, UMR 5209 CNRS-Université de Bourgogne, 49 Rue Mademoiselle Bourgeois, \\ 58027 Nevers Cedex, France \\ Philippe Leclaire \\ Département de Recherche en Ingénierie des Véhicules pour l'Environnement, ISAT, Université de Bourgogne, \\ 49 Rue Mademoiselle Bourgeois, 58027 Nevers Cedex, France
}

(Received 7 February 2012; revised 14 May 2012; accepted 28 August 2012)

\begin{abstract}
A hybrid model describing the acoustic properties of plates with macroperforations that can be unevenly distributed on the plate surface and backed by woven or precision woven meshes with microscopic perforations is proposed. The plate perforations may be of circular or rectangular shapes. Since the perforated plate may not necessarily be considered as an equivalent fluid, its impedance is calculated by the Maa model [Noise Control Eng. J. 29, 77-84 (1987)], whereas the JohnsonChampoux-Allard model [J. Appl. Phys. 70, 1975-1979 (1991)] is used for the mesh, considered as an equivalent fluid. A simple model for the elementary cell of the mesh structure is proposed in order to calculate parameters that can be considered as the thermal characteristic length $\Lambda^{\prime}$ and the viscous characteristic length $\Lambda$. An effective airflow resistivity is introduced to account for the increase of particle velocity through the mesh placed behind the carrying macroperforated plate and is used in the transfer matrix approach to obtain the impedance of the whole multilayer system. The hybrid model seems to represent a good approach of this multilayer system. The theoretical predictions are compared with experimental measurements.
\end{abstract}

(C) 2012 Acoustical Society of America. [http://dx.doi.org/10.1121/1.4754520]

PACS number(s): 43.55.Ev, 43.50.Gf, 43.20.Hq [KVH]

Pages: $3138-3147$

\section{INTRODUCTION}

The acoustical behavior of perforated panels has been an important research topic over the last decades. The analytical model proposed by $\mathrm{Maa}^{1}$ is frequently used to predict the acoustic impedance of a microperforated panel (MPP) with circular perforations. The equation for the impedance combines the model of sound propagation in narrow tubes studied by Rayleigh and Crandall ${ }^{2,3}$ and includes the end corrections proposed by Ingard. ${ }^{4}$ In the useful audible frequency range, the best performance for perforated panels is obtained for submillimetric holes. As recommended by Maa, ${ }^{1}$ the diameter of perforation-to-thickness ratio should be close to one to obtain optimal absorption properties. Manufacturing panels with submillimetric holes and submillimetric thicknesses can be a difficult task in practical acoustic engineering applications. Furthermore, drilling a large amount of submillimetric holes in a panel (usually done by laser technology) can be expensive. An alternative method to reduce the amount of perforations while keeping the

\footnotetext{
a) Author to whom correspondence should be addressed. Electronic mail: heidi@caend.upm-csic.es
}

porosity equal to that of an MPP is to use slits. ${ }^{5,6}$ Manufacturing a panel bearing slits (MSP) can be less expensive than manufacturing an MPP.

A perforated plate can, in some cases, also be modeled as a rigid frame porous medium described by the well established Johnson-Champoux-Allard (JCA) theory ${ }^{7-9}$ that depends on five physical parameters: the thermal $\Lambda^{\prime}$ and viscous $\Lambda$ characteristic lengths, the porosity, $\phi$, the tortuosity, $\alpha_{\infty}$, and the static air flow resistivity, $\sigma$. Atalla and Sgard ${ }^{10}$ presented an equivalent fluid approach that considers these parameters. The analytical models of Maa and the equivalent fluid approach agree in what concerns the impedance inside the perforations. Nevertheless, the end corrections differ. While $\mathrm{Maa}^{1,5}$ introduces the end correction as an additional complex impedance with resistive and reactive components, Atalla and Sgard ${ }^{10}$ introduce the end correction by means of the geometric tortuosity, $\alpha_{\infty}$, as a multiplying factor of the effective density, $\tilde{\rho}_{e}$.

To increase the sound attenuation, perforated panels can be backed by porous materials or textiles. ${ }^{11-13}$ Combining a carrying plate with a commercial woven mesh makes it possible to design an absorber with a performance similar to that of a single-layer MPP. Indeed, Pfretzschner et al. ${ }^{12}$ proposed a 
design procedure that consisted on combining a perforated panel and a micrometric mesh with appropriate constitutive parameters, which they named a microperforated insertion unit (MIU). The impedance of the MIU resulted from the addition of the acoustic impedances of the perforated plate and of the wire mesh divided by the open area fraction of the plate $\phi$. The impedance of the system was then obtained by the impedance transfer method. ${ }^{14}$

Recently, Chevillote ${ }^{13}$ studied the role of a resistive layer upstream on a porous medium. The resistive layer was modeled using the JCA equivalent fluid model. ${ }^{7-9}$ The acoustic impedance of such multilayer system was obtained using the transfer matrix method. ${ }^{7}$ Chevillote used a twodimensional microscopic characterization with help of a picture processing to determine the hydraulic radius and deduce the thermal characteristic length of the textile.

In this article, we study a multilayer system made of macroperforated metal plates backed by microperforated woven textile meshes. This solution involving fairly high perforation radii for the carrying plate and widely available textiles can significantly decrease the costs of manufacturing of the acoustic system. The prefixes "micro" or "macro" are related to the relative importance of the radius with respect to the viscous skin depth inside the perforation.

In the present study, a new approach involving a simple model for the elementary cell of the woven textile structure is proposed to estimate more precisely the thermal and viscous characteristic lengths. Also, a hybrid model capable of describing non-homogenously distributed macroperforated panels backed by woven textiles is proposed. The carrying plate is modeled using the analytical approach ${ }^{1,5}$ of Maa, whereas the JCA model is used in the rigid frame approximation for the mesh. The hybrid model is valid for carrying panels with circular or slitlike macroperforations regardless of their distribution over the surface. Another original aspect developed in this article is the introduction of an effective resistivity. The permeability (of the flow resistivity) of the mesh is calculated by means of the shape factor described by Stinson and Champoux. ${ }^{15}$ In addition, whereas in Ref. 12 the fact that bonding the membrane to the carrying plate modified the perforation rate of the screen, it is shown in the present study that the modification can be accounted for through the use of an effective resistivity.

The description of the theory used to obtain the impedance of the carrying plate is presented in Sec. II. The calculation of the thermal characteristic length of microperforated woven meshes is described in Sec. III. Section IV presents the hybrid model using a combination of the models of Maa and JCA in the context of the transfer matrix method and also the derivation of the effective flow-resistivity. Experimental validation is presented in Sec. $\mathrm{V}$.

\section{PERFORATED PLATE}

The impedance of a perforated panel can be written as the sum of two contributions: the impedance at the interior of the perforation and the impedance at the borders which accounts for the end corrections. For a plate perforated with circular perforations, Maa obtained the following equation: ${ }^{1}$

$$
Z_{1 \mathrm{MPP}}=\frac{\sqrt{2} \mu y}{\phi_{1} d_{1}}+\frac{j \omega \rho_{0}}{\phi_{1}}\left[\frac{0.85 d_{1}}{\psi(\xi)}+\frac{t_{1}}{\left[1-\frac{2}{y \sqrt{-j}} \frac{J_{1}(y \sqrt{-j})}{J_{0}(\sqrt{-j})}\right]}\right]
$$

where $\mu$ is the air dynamic viscosity coefficient, $\rho_{0}$ is the air density, $\omega$ is the angular frequency, $t_{1}$ is the panel thickness, $\phi_{1}$ is the perforation ratio, $J_{0}, J_{1}$ are Bessel functions of the first kind and of order 0 and 1 , respectively, and $y=d_{1} /$ $\sqrt{4 \mu / \rho_{0} \omega}$ with $d_{1}$ the perforation diameter. A similar equation but accounting for slitlike perforations yields ${ }^{5}$

$$
Z_{1 \mathrm{MSP}}=\frac{\sqrt{2} \mu y}{\phi_{1} d_{1}}+\frac{j \omega \rho_{0}}{\phi_{1}}\left[\frac{F_{(e)}}{\psi(\xi)} \frac{d_{1}}{2}+\frac{t_{1}}{\left[1-\frac{\tanh (y \sqrt{j})}{y \sqrt{j}}\right]}\right],
$$

where $F_{(e)}$ is an elliptic integral with $e=\sqrt{1-\left(r_{1} / l_{1}\right)^{2}}$, where $l_{1}$ denotes the length of the slit and $r_{1}$ is the perforation radius,

$$
\begin{aligned}
F_{(e)}= & \frac{\pi}{2}\left[1+\left(\frac{1}{2}\right)^{2} e^{2}+\left(\frac{1 \times 3}{2 \times 4}\right)^{2} e^{4}\right. \\
& \left.+\left(\frac{1 \times 3 \times 5}{2 \times 4 \times 6}\right)^{2} e^{6}+\cdots\right] .
\end{aligned}
$$

Equations (1) and (2) were obtained by Maa as follows: The end corrections studied by Rayleigh and Ingard ${ }^{2,4}$ are included in the first and second terms. The influence of a mass of air contained in a cylindrical volume for which the apparent length is greater than the plate thickness is included in the numerator of the second term. The third term represents the impedance of the perforations which has been studied by Crandall $^{3}$ for an array of tubes, and by Ingard $^{4}$ for an elliptic aperture. The velocity profile of the flow through the holes is determined by the term $y$.

The over perforation effect is included in this work for the case of periodic hole arrangement by means of the term $\psi(\xi)$, which is a correction of the reactance term of the end correction that takes into account the interaction between holes when the perforation ratio is high. ${ }^{16,17} \psi(\xi)=1 /(1-1.4092(\xi)$ $+0.33818(\xi)^{3}+0.06791(\xi)^{5}-0.02287(\xi)^{6}+0.03015(\xi)^{7}$ $\left.-0.01641(\xi)^{8}\right)$, with $\xi=0.88 d_{1} / g_{1}$. The term $g_{1}$ is the distance between the holes centers.

The classical MPP's formulation ${ }^{18}$ and the equivalent fluid MPP's approach ${ }^{10}$ take into account the increase of kinetic energy caused by the sound radiation effect at the end openings of perforations and the distortion effect of the acoustic flow at the panel surface. As a result, the MPP's reactance must be supplemented by an additional mass by way of the length correction on the plate thickness, in the imaginary part of the MPP's impedance. ${ }^{4,7}$ In the case of a single perforation, the classical approach employs an analogy with the radiation impedance of a rigid piston with a circular cross section flanged in an infinite rigid wall. For this approach the end termination of perforation is considered to have a sharp edge. 
The interaction between slit perforations together with the resistive and reactive end corrections deduced for the modified depth of the slit, ${ }^{19}$ was as well described by Randeberg in Ref. 6 . However, for the present study the equations proposed by Maa will be used to model a macroperforated carrying plate.

\section{MICROMETRIC MESH}

The absorption of textiles can be calculated following different models. For instance, Kang and Fuchs determined the absorption of microperforated membranes using the equation of impedance given by Maa. In a recent study, Atalla and Sgard considered a plate or screen as an equivalent fluid using the rigid frame porous model developed by JohnsonChampoux-Allard. $^{7-9}$ In the equivalent fluid approach, the end corrections are introduced by means of the tortuosity allowing it to define the boundary conditions in multilayer configurations. Latest works apply this model for woven or non-woven fabrics. ${ }^{13,20}$ In many cases, a homogeneous cross section of perforations is considered (for example, cylindrical or square cross section). Nevertheless, as the woven meshes studied here exhibit more complex pore geometries, a more specific approach is needed (three dimensions approach is required for some parameter characterizations).

In the JCA model, the tortuosity, resistivity, porosity, and characteristic lengths of the pores can be obtained ${ }^{8}$ or measured for each specific equivalent fluid. The viscothermal effects associated with a wave impinging onto a pore of a porous medium can be modeled introducing a complex dynamic density, i.e., the effective density $\tilde{\rho}_{e}(\omega)$ (see Ref. 7)

$$
\tilde{\rho}_{e}(\omega)=\alpha_{\infty} \rho_{0}\left(1+\frac{\sigma_{2} \phi_{2}}{j \omega \rho_{0} \alpha_{\infty}} \sqrt{1+\frac{j \omega 4 \alpha_{\infty}^{2} \mu \rho_{0}}{\sigma^{2} \Lambda^{2} \phi_{2}^{2}}}\right),
$$

where $\alpha_{\infty}$ is the geometrical tortuosity, $\rho_{0}$ is the air density, $\sigma_{2}$ is the flow resistivity, $\phi_{2}$ is the porosity, which is considered equivalent to the perforation ratio, $\omega$ is the angular frequency, $\mu$ is the air dynamic viscosity coefficient, and $\Lambda$ is the viscous length given by ${ }^{8}$

$$
\frac{2}{\Lambda}=\frac{\int_{S} \vartheta_{i}^{2}\left(r_{w}\right) d S}{\int_{V} \vartheta_{i}^{2}(r) d V},
$$

where $\vartheta_{i}\left(r_{w}\right)$ is the velocity of an inviscid fluid at the surface of the pore and $\vartheta_{i}(r)$ is the velocity inside the pore, assuming static flow. The integrations of the numerator and the denominator of Eq. (5) are made over the pore surface, $S$, and over the pore volume, $V$, respectively.

Equation (4) indicates that the parameters $\alpha_{\infty}$ and $\sigma_{2}$ are important factors in the calculation of the effective density. While the flow resistivity depends on the pore shape, the geometrical tortuosity depends on the pore shape and the layer arrangement. For a screen with air at both sides, $\alpha_{\infty}=1+\left(2 \varepsilon_{2} / t_{2}\right)$, where $\varepsilon_{2}$ represents a correction length and $t_{2}$ is the thickness of the mesh. ${ }^{10}$
In the present study, the end termination of the screen perforation has rounded edges. This kind of edge tends to diminish the length correction. ${ }^{21}$ Moreover, when there are several perforations in close proximity, one must consider that the interaction between perforations tends to diminish the attached mass. ${ }^{4,6}$ For the meshes treated in this study, the flange between two perforations is small compared to their size, which causes a strong interaction between the rounded edges of the pore. The length correction is thought to be very small and is therefore disregarded. As a result, the effective tortuosity is taken to be equal to 1 .

The flow resistivity, $\sigma_{2}$, has been described by Stinson and Champoux ${ }^{15}$ for simple pore shapes as

$$
\sigma_{2}=\frac{4 q_{0} \mu \alpha_{\infty}^{2}}{h_{r}^{2} \phi_{2}}
$$

where $h_{r}$ is the hydraulic radius defined as two times the quotient of the cross sectional area to the circumference of the pore, ${ }^{15}$ and $q_{0}$ is a constant parameter for a given pore shape. When the perforations are assumed to be of cylindrical shape with constant cross section, the viscous characteristic length and the thermal characteristic length are equal to the hydraulic radius of the perforation, ${ }^{7,10,13,20} \Lambda=\Lambda^{\prime}=h_{r}=d_{2} / 2$ $=r_{2}$. However, as will be seen in Sec. IV, this assumption cannot be made for other pore geometries.

Considering woven membranes as materials with identical uniform pores, for each pore the effective density can be linked to the dynamic bulk modulus given by ${ }^{7,9}$

$$
K(\omega)=\frac{\gamma P_{0}}{\gamma-(\gamma-1)(1+H \sqrt{1+j \omega / H} / j 2 \omega)},
$$

where $\gamma$ is the fluid specific heat ratio, $P_{0}$ is the fluid equilibrium pressure, $\omega$ the angular frequency, and $H=16 \mu$ / $\left(N_{\operatorname{Pr}} \Lambda^{\prime 2} \rho_{0}\right) . N_{\operatorname{Pr}}$ is the Prandtl number. The thermal length $\Lambda^{\prime}$ characterizes the high frequency behavior of the bulk modulus. ${ }^{9}$ A simple model to calculate $\Lambda^{\prime}$ is presented in Sec. III A.

Equations (4) and (7) allow us to calculate the characteristic impedance $Z_{c 2}$ of the fluid

$$
Z_{c 2}=\sqrt{K(\omega) \times \tilde{\rho}_{e}(\omega)}
$$

and the associated wave number

$$
k_{2}=\omega \sqrt{\tilde{\rho}_{e}(\omega) / K(\omega)}
$$

\section{A. Simple model for calculating the thermal length of woven meshes}

The thermal characteristic length has been defined as the surface to pore volume ratio of the pore solid interface ${ }^{9}$

$$
\frac{2}{\Lambda^{\prime}}=\frac{\int_{S} d S}{\int_{V} d V}=\frac{S}{V} .
$$




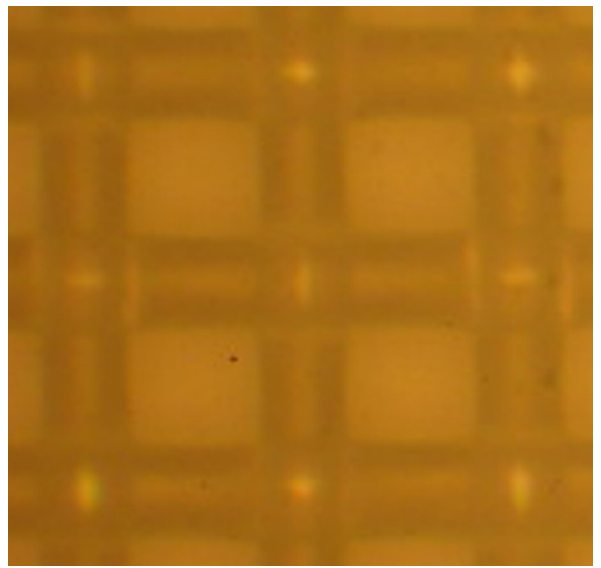

FIG. 1. (Color online) Microscopic image of an elementary cell of a woven mesh.

The volume of air traveling through the pore depends on the end termination of the yarn geometry. Assuming isotropic meshes with cylindrical yarns, an original model to obtain the thermal length is proposed. The elementary cell of the screen perforation is shown in Fig. 1. A straightforward calculation can be made by observing a single perforation as the one in Fig. 2(a). The volume of air through the cell is observed in Fig. 2(b). The volume of each truncated cylinder in the elementary cell of Fig. 2(a) is

$$
V_{h}=\pi r_{y}{ }^{2} b-4 \int_{x=-r_{y}}^{r_{y}} \int_{y=0}^{\sqrt{r_{y}^{2}-x^{2}}} \int_{z=0}^{y} d x d y d z
$$

where $r_{y}$ is the yarn radius and $b=d_{2}+2 r_{y}$. Solving the integral of the second term on the right hand side of Eq. (11) gives

$$
V_{h}=\pi r_{y}{ }^{2} b-4 \frac{2 r_{y}^{3}}{3} .
$$

The volume, $V_{p}$, of the pore [Fig. 2(b)] is then

$$
V_{p}=b^{2} Y_{d}-\frac{4 V_{h}}{2}
$$

Figure 2(b) represents Eq. (13). The surface of each truncated cylinder is

(a)

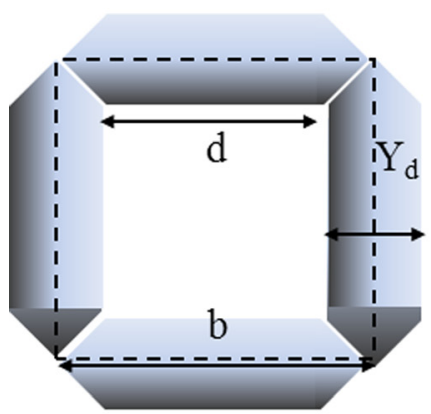

$$
S_{h}=2 \pi r_{y} b-4 \int_{0}^{\pi} r_{y}^{2} \sin \theta d \theta
$$

Solving the integral in Eq. (14) we obtain

$$
S_{h}=2 \pi r_{y} b-8 r_{y}^{2} \text {. }
$$

The interior surface, $S_{p}$, of the pore is then

$$
S_{p}=\frac{4 S_{h}}{2} .
$$

The thermal length can be obtained by replacing $S$ with $S_{p}$ and $V$ with $V_{p}$ in Eq. (10)

$$
\Lambda^{\prime}=\frac{b^{2}-b \pi r_{y}+(8 / 3) r_{y}^{2}}{\pi b-4 r_{y}} .
$$

The calculation of the viscous characteristic length, $\Lambda$, using the finite element method is proposed in the Appendix. The assumptions considered are clearly stated in the Appendix and are those of the definition of $\Lambda$. The calculated values of $\Lambda^{\prime}$ [Eq. (17)] and of $\Lambda$ were used in the numerical simulations of the absorption coefficient. Assuming identical pores for the particular geometry of a square pore of woven meshes, $\alpha_{\infty}=1, \sigma_{2}$ is obtained with Eq. (6) where wh $^{15}$ $q_{0}=1.78$ and $\phi_{2}=\left(d_{2} /\left(Y_{d}+d_{2}\right)\right)^{2}$.

\section{THE HYBRID MODEL AND THE TRANSFER MATRIX APPROACH}

A hybrid model combining the Maa and the JCA model to obtain the input impedance of a system composed of a macroperforated plate and a micrometric commercial woven mesh as in Fig. 3 is proposed.

As only the upstream side of the plate is free, the reactive term of the impedance [Eqs. (1) and (2)] should be multiplied by 0.5 . The presence of the screen will modify the flow distortion at the perforations' end termination close to the screen, thus the end correction length of the MPP(MSP) reactance must be corrected. In the case of perforated plates in contact with porous layers, Atalla and Sgard ${ }^{10}$ proposed to take into account the distortion of the flow and to define the MPP(MSP) end correction length for the side in contact with the porous material as $\varepsilon_{1}(\operatorname{Re}(\alpha(\omega))) . \alpha(\omega)$ is the dynamic tortuosity of the mesh and $\varepsilon_{1}=0.48 \sqrt{A_{\text {perf }}}\left(1-1.47 \sqrt{\phi_{1}}\right.$

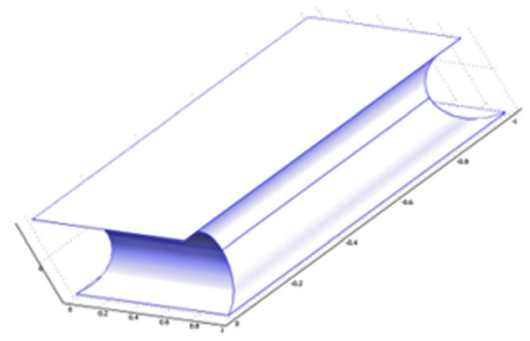

FIG. 2. (Color online) Simple model for a single mesh perforation geometry. (a) Fiberstructure (the dotted rectangle represents the elementary cell), (b) inside volume. 


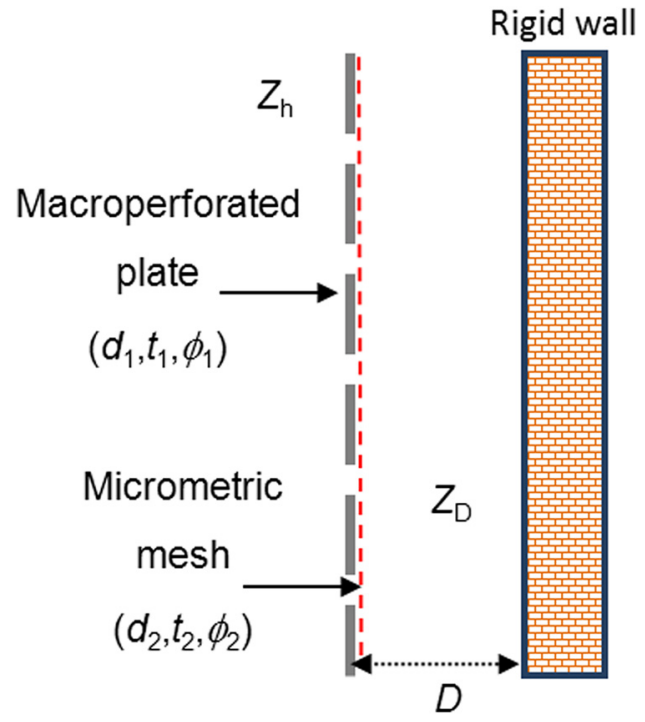

FIG. 3. (Color online) Hybrid model consisting of a woven mesh with $\left(d_{1}, t_{1}, \phi_{1}\right)$ and a woven mesh with $\left(d_{2}, t_{2}, \phi_{2}\right)$ in front of a rigid wall with an air cavity thickness $D$.

$\left.+0.47 \sqrt{\phi_{1}^{3}}\right)$ is the correction length of the MPP with $A_{\text {perf }}=\pi r_{1}^{2}$ for circular perforations and $A_{\text {perf }}=d_{1} l_{1}$ for slits. Atalla and Sgard discussed the possibility of substituting $\operatorname{Re}(\alpha(\omega))$ by $\alpha_{\infty}$ in the end correction. However, they noted that for an MPP(MSP) in contact with a rigid frame porous material, the comparison with experimental results was better with $\operatorname{Re}(\alpha(\omega))$. In the present study, for an MPP(MSP) in contact with a screen, the choice of $\alpha_{\infty}$ gives better results. Equations (1) and (2) corrected for the hybrid model are rewritten in Eqs. (18) and (19), respectively,

$$
\begin{aligned}
\mathrm{Z}_{1 \mathrm{MPP}}= & \frac{\sqrt{2} \mu \mathrm{y}}{\phi_{1} \mathrm{~d}_{1}}+\frac{\mathrm{j} \omega \rho_{0}}{\phi_{1}}\left[\frac{0.85 \mathrm{~d}_{1}}{\psi(\xi)} \times 0.5+\varepsilon_{1}\left(\alpha_{\infty}\right)\right. \\
& \left.+\frac{t_{1}}{\left[1-\frac{2}{y \sqrt{-j}} \frac{J_{1}(y \sqrt{-j})}{J_{0}(y \sqrt{-j})}\right]}\right]
\end{aligned}
$$

and

$$
\begin{aligned}
\mathrm{Z}_{1 \mathrm{MSP}}= & \frac{\sqrt{2} \mu y}{\phi_{1} d_{1}}+\frac{j \omega \rho_{0}}{\phi_{1}}\left[\frac{F_{(e)} d_{1}}{2 \psi(\xi)} \times 0.5+\varepsilon_{1}\left(\alpha_{\infty}\right)\right. \\
& \left.+\frac{t_{1}}{\left[1-\frac{\tanh (y \sqrt{j})}{y \sqrt{j}}\right]}\right]
\end{aligned}
$$

Note that since the effective static tortuosity of the mesh is equal to 1 , the end correction of the MPP(MSP) in contact with the mesh is similar to the end correction of an MPP(MSP) in air.

The absorption curve of the hybrid system depends on seven parameters $\left(d_{1}, d_{2}, t_{1}, t_{2}, \phi_{1}, \phi_{2}, D\right)$ which are the pore diameter, thickness, and porosity of the carrying plate and the mesh, respectively, and $D$ is the air cavity thickness. In

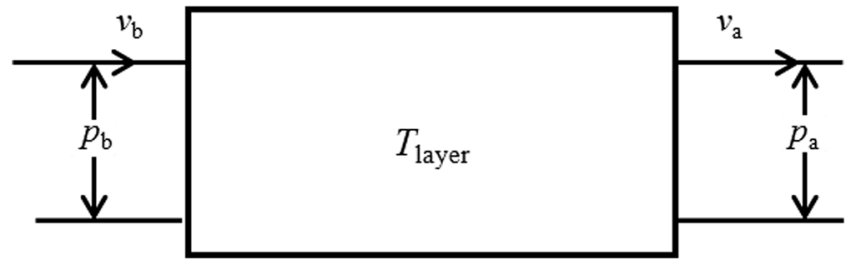

FIG. 4. One-dimensional acoustic element of four pole parameters.

the work of Pfretzschner et al., the impedance of a system composed of a perforated plate carrying a micrometric mesh was obtained using the impedance transfer method. ${ }^{12,14} \mathrm{Kang}$ and Fuchs ${ }^{11}$ used the electrical circuit approach for the same purpose. In the present work, the transfer matrix approach ${ }^{7}$ is used and the acoustic impedance of the whole system is obtained considering the unit transfer matrix of each layer as

$$
\left[\begin{array}{c}
p_{b} \\
v_{b}
\end{array}\right]=T_{\text {layer }}\left[\begin{array}{c}
p_{a} \\
v_{a}
\end{array}\right]
$$

where $p$ and $v$ are the pressure and particle velocity at the upstream, $b$, and downstream, $a$, sides $^{22}$ (see Fig. 4). The transfer matrix can be generalized for a $4 \times 4$ matrix (threedimensional case), but since the comparison with experimental measurements at normal sound incidence was carried out, only the $2 \times 2$ matrix was used. Here, $T_{\text {layer }}$ represents a onedimensional acoustic element. The hybrid model consists of three layers $T_{1}, T_{2}$, and $T_{3}$, i.e., $T_{\text {Plate }}, T_{\text {Mesh }}$, and $T_{\text {Air }}$.

Using Eq. (18) or (19), the impedance transfer matrix for a carrying plate with circular or slits perforations can be obtained as

$$
T_{1}=T_{\text {Plate }}=\left[\begin{array}{ll}
1 & Z_{1} \\
0 & 1
\end{array}\right]
$$

In this study, carrying plates with perforation sizes greater than $1 \mathrm{~mm}$ and perforations rates close to $10 \%$ are considered, the viscous and thermal characteristic frequency is very low, so the thermo-viscous effect can be disregarded, ${ }^{7}$ however, the mass effect is very high. As a consequence the impedance expression can be reduced in such a way that only the imaginary part is taken into account and Eq. (21) can be rewritten as

$$
T_{1}=T_{\text {Plate }}=\left[\begin{array}{cc}
1 & j\left(\operatorname{imag}\left(Z_{1}\right)\right) \\
0 & 1
\end{array}\right] .
$$

Indeed, the experimental sound absorption coefficient of this plate (MPP or MSP without screen) coupled with an air cavity and rigid wall is weak.

The transfer matrix for a mesh in the equivalent fluid context is given by

$$
T_{2}=T_{\text {Mesh }}=\left[\begin{array}{cc}
\cos \left(k_{2} t_{2}\right) & j Z_{c 2} \sin \left(k_{2} t_{2}\right) / \phi_{2} \\
j \phi_{2} \sin \left(k_{2} t_{2}\right) / Z_{c 2} & \cos \left(k_{2} t_{2}\right)
\end{array}\right],
$$

where $t_{2}, \phi_{2}, Z_{c 2}, k_{2}$ are the thickness, the porosity, the characteristic impedance, and the wave number of the fluid in the mesh, respectively. Note that in order to include the boundary conditions outside the mesh, the impedance $Z_{c 2}$ in Eq. (23), is 
being divided by the porosity of the mesh $\phi_{2}$. In this way the impedance obtained with Eq. (8) for an equivalent fluid is specified for the equivalent material. $k_{2}$ can be obtained with Eq. (9).

For the air cavity, $t_{2}, \phi_{2}, Z_{c 2}, k_{2}$ are replaced by the properties of air, i.e., $D, 1, Z_{0}=\rho_{0} c_{0}$, (where $c_{0}$ is the velocity of sound in air) and $k_{0}=2 \pi f$ which represent the thickness, the porosity, the characteristic impedance, and the wave number of the air in the cavity. The corresponding matrix is

$$
T_{3}=T_{\text {Air }}=\left[\begin{array}{cc}
\cos \left(k_{0} D\right) & j Z_{0} \sin \left(k_{0} D\right) \\
j \sin \left(k_{0} D\right) / Z_{0} & \cos \left(k_{0} D\right)
\end{array}\right] .
$$

The multiplication of the individual matrices results in the global transfer matrix of the hybrid model

$$
T_{h}=\left[T_{\text {Plate }}\right]\left[T_{\text {Mesh }}\right]\left[T_{\text {Air }}\right] .
$$

A transfer matrix can be adapted for each new layer of plate, mesh, or air. The input impedance of the system and absorption coefficient are then obtained by

$$
\begin{aligned}
& Z_{h}=\frac{T_{h 11}}{T_{h 21}}, \\
& \alpha=1-\left|\frac{Z_{h}-Z_{0}}{Z_{h}+Z_{0}}\right|^{2} .
\end{aligned}
$$

The schematic represented in Fig. 5 shows the effect of the flow inside the perforations. The particle velocity $\vartheta$ is the same at both sides of the hybrid system. As $\phi_{1}$ and $\phi_{2}$ are always smaller than 1 , the particle velocity inside the pores is always greater than outside them. The relationship between the pressure gradient $\Delta P$ and the particle velocity $\vartheta$ is described by Darcy's law

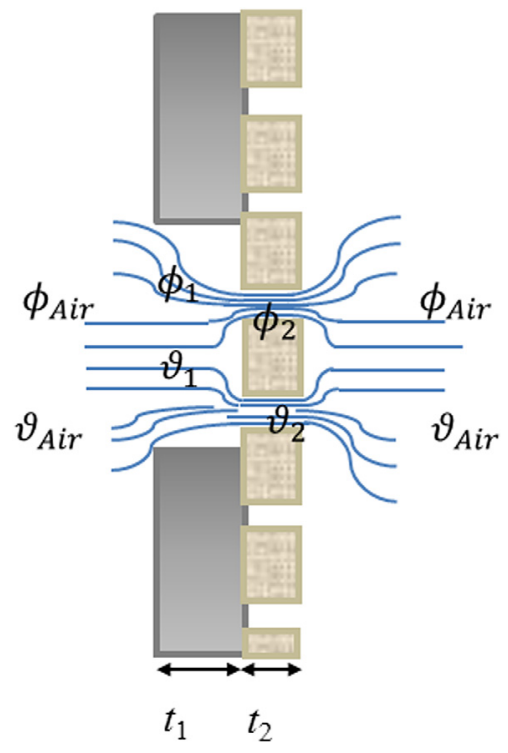

FIG. 5. (Color online) Viscous flow of the fluid through the perforations. Downstream the perforation, the "apparent" fluid velocity in the mesh is increased because of the squeezing due to the presence of the perforations upstream. This results in an "effective flow resistivity" that accounts for this diaphragm effect.

$$
\Delta P=-\phi \vartheta \sigma
$$

where $\phi, \vartheta$, and $\sigma$ are the porosity, normal velocity of the fluid through the system, and resistivity. This equation can be used to interpret the flow in the woven mesh. However, when a macroperforated plate is coupled with a mesh without airspace between the two elements, a correction should be applied to this law to incorporate a "diaphragm effect" (Fig. 5). As the flow is squeezed inside the plate macroperforations, the flow velocity at the entrance of the pores of the mesh is increased and the flow resistivity of the mesh should be modified to account for this phenomenon. The correction factor on the flow resistivity is precisely the porosity of the carrying plate. As a result, the resistivity of the mesh should be replaced by an "effective resistivity" of the combination plate-mesh

$$
\sigma_{\mathrm{eff}}=\frac{\sigma_{2}}{\phi_{1}}
$$

Therefore, the modified Darcy law for the mesh is

$$
\Delta P_{2}=-\phi_{2} \vartheta_{2} \sigma_{\text {eff }}=-\frac{\phi_{2}}{\phi_{1}} \vartheta_{2} \sigma_{2} .
$$

This correction is to be made in Eq. (4), where $\sigma_{\text {eff }}$ should be used instead of $\sigma_{2}$. The effect of modifying the mesh resistivity by the effective resistivity is shown in Fig. 6. For perforated facings, the resistivity is inversely proportional to the porosity, ${ }^{7}$ thus the introduction of the effective resistivity can be considered as equivalent to the change in the porosity in the work of Pfretzschner et al. ${ }^{12}$

\section{EXPERIMENTAL RESULTS}

The theory exposed previously is now confronted to experimental results at normal incidence sound absorption with plane wave propagation. The validity of the approaches used to evaluate $\Lambda^{\prime}$ and $\sigma_{2}$ is tested. The hybrid model is used on combinations of four different macroperforated panels used

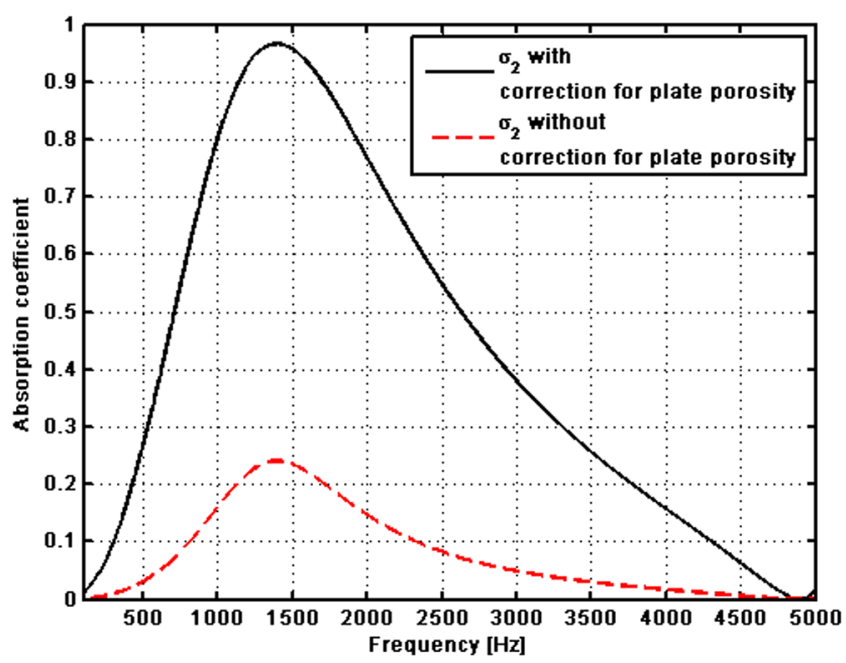

FIG. 6. (Color online) Normal incidence sound absorption coefficient of a carrying plate with $\left(d_{1}, t_{1}, \phi_{1}\right)=(3 \mathrm{~mm}, 1 \mathrm{~mm}, 10 \%)$ with a micrometric mesh $\left(d_{2}, t_{2}, \phi_{2}\right)=(50 \mu \mathrm{m}, 50 \mu \mathrm{m}, 37 \%)$ and $D=3.5 \mathrm{~cm}$. (-) with correction for porosity, (---) without correction. 


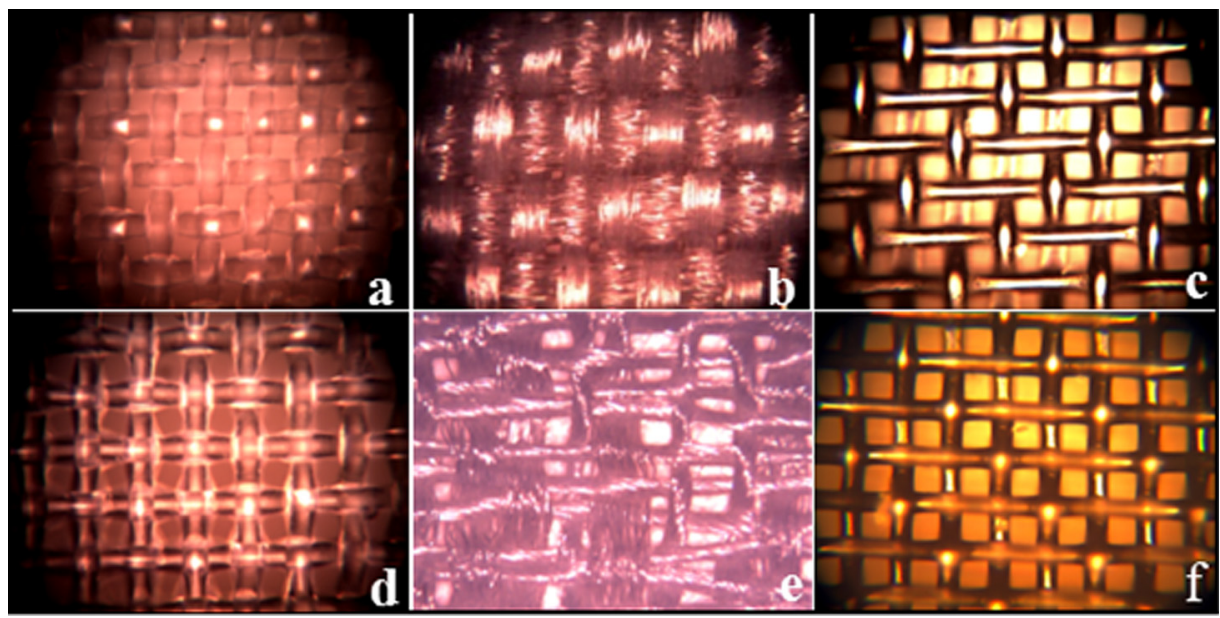

FIG. 7. (Color online) Microscopic image of the meshes tested. (a) Sefar nitex $36 / 28$, (b) woven screen, (c) Sefar acoustic BHY, (d) Sefar nitex 41/31, (e) curtain, and (f) Sefar acoustic HF 34-39.

as carrying plates for five precision and non-precision woven meshes.

A Bruel \& Kjaer (Naerum, Denmark) impedance tube type 4206 with inner diameter of $29 \mathrm{~mm}$ was used. Samples of $28.8 \mathrm{~mm}$ diameter were constructed and mounted inside the tube, parallel to the rigid termination. An air gap separated the sample from the tube's termination. To assure perfect sealing and vertical positioning, plasticine was used at the edges of the samples. Special attention was made while positioning the sample in the tube in order to minimize the errors in the measurement. The transfer function between two Bruel \& Kjaer type 2670 microphones separated by $20 \mathrm{~mm}$ was measured. The smallest distance between the samples and the microphones was $15 \mathrm{~mm}$. The absorption coefficient was calculated using the transfer function method. ${ }^{23}$ The laboratory atmospheric conditions were taken into account to define the air constants.

Precision woven and non-precision woven meshes where used. Figure 7 shows the six meshes used. The pores and yarn diameter were obtained using the software Optika vision lite and an Euromex Holland microscope. The microscopic analysis allowed us to calculate the thermal and viscous characteristic lengths. The thickness was measured with a digital caliper. The porosity was evaluated by the expression $\phi_{2}=\left(d_{2} /\left(Y_{d}+d_{2}\right)\right)^{2}$. The precision on the evaluation of the porosity when compared to the manufacturer's value was $5 \%$. Results are shown in Table I.

To observe the acoustic behavior of a micrometric mesh, a single mesh was measured in the impedance tube without a carrying panel. Figure 8 shows the absorption curve of the commercial woven mesh Sefar (Heiden, Switzerland) nitex 36/28 (mesh $a$ in Table I) with $\left(d_{2}, t_{2}, \phi_{2}, Y_{d}, D\right)=(36.38 \mu \mathrm{m}, 50 \mu \mathrm{m}$, $27.23 \%, 33.34 \mu \mathrm{m}, 5 \mathrm{~cm}$ ), where $d_{2}$ is the diameter of perforations, $t_{2}$ is the thickness, $\phi_{2}$ is the perforation ratio, $Y_{d}$ is the yarn diameter, and $D$ is the air cavity thickness between the mesh and the tube termination. A good agreement between the JCA model and the measurement is achieved. The real and imaginary parts of the dynamic tortuosity and bulk modulus of air in the mesh are observed in Fig. 9. The frequency dependence of these two quantities is clearly seen. At high frequencies the bulk modulus increases indicating an adiabatic process. At low frequencies, an isothermal process is observed in the real part of the bulk modulus which approaches the value of one asymptotically.

The experimental validation of the hybrid model was then carried out. The meshes were bonded to the portable panels by means of glue. Figure 10 shows the four different macroperforated carrying plates with $d_{1}=3 \mathrm{~mm}, \phi_{1}=9.96 \%$, and $t_{1}=1 \mathrm{~mm}$. In plates 1,2 , and 4 the hydraulic diameter is equal to $r_{h}=\Lambda^{\prime}=l_{1} d_{1} /\left(l_{1}+d_{1}\right)$, while in plate $3 r_{h}=d_{1} / 2$ $=r_{1}$ as in Ref. 15. A good adjustment is obtained for $\xi=0.25,0.25,0.35$, and 0.28 for plates 1 through 4 , respectively. Figure 11 illustrates the results of combining plate 1 with meshes $b, c$, and $e$ (woven screen, Sefar acoustic BHY 34-39, and curtain, respectively). Two interesting results are highlighted. One on hand, it is observed that the hybrid model presents a very good agreement with the experimental measurements for precision woven and non-precision woven meshes. On the other hand, a very high absorption is achieved by using mesh $c$ and $e$, and while the first one is a commercial precision woven mesh used for filtering, the second one is a simple woven curtain. The difference in costs

TABLE I. Characteristics of the meshes.

\begin{tabular}{|c|c|c|c|c|c|c|c|c|c|c|}
\hline Mesh & reference & $\begin{array}{c}d_{2} \\
(\mu \mathrm{m})\end{array}$ & $\begin{array}{c}t_{2} \\
(\mu \mathrm{m})\end{array}$ & $\begin{array}{c}\phi_{2} \\
(\%)\end{array}$ & $\begin{array}{c}Y_{d} \\
(\mu \mathrm{m})\end{array}$ & $\begin{array}{c}\Lambda \\
(\mu \mathrm{m})\end{array}$ & $\begin{array}{c}\Lambda^{\prime} \\
(\mu \mathrm{m})\end{array}$ & $\Lambda / \Lambda^{\prime}$ & $\begin{array}{c}\sigma \\
\left(\mathrm{Nsm}^{-4}\right)\end{array}$ & $\begin{array}{c}\sigma_{\text {eff }} \\
\left(\mathrm{Nsm}^{-4}\right)\end{array}$ \\
\hline$a$ & Sefar nitex $36 / 28$ & 36.38 & 50 & 27.23 & 33.34 & 14,00 & 12,40 & 1,126 & $1.45 \times 10^{6}$ & Not used \\
\hline$b$ & Woven screen & 59.18 & 110 & 5.29 & 198.21 & 33,60 & 30,00 & 1,122 & $2.82 \times 10^{6}$ & $2.83 \times 10^{7}$ \\
\hline$c$ & Sefar acoustic BHY 34-39 & 39 & 49 & 34.9 & 33 & 15,03 & 13,60 & 1,105 & $9.84 \times 10^{5}$ & $9.88 \times 10^{6}$ \\
\hline$d$ & Sefar nitex $41 / 31$ & 45.7 & 45.87 & 36.35 & 30.1 & 16,05 & 15,50 & 1,032 & $6.85 \times 10^{5}$ & $6.92 \times 10^{6}$ \\
\hline$e$ & Curtain & 94.25 & 120 & 19.7 & 117.8 & 41,60 & 34,80 & 1,194 & $2.97 \times 10^{5}$ & $2.99 \times 10^{6}$ \\
\hline$f$ & Sefar acoustic HF 34-39 & 39 & 45.37 & 34.90 & 30.29 & 14,57 & 13,48 & 1,081 & $9.84 \times 10^{5}$ & $9.88 \times 10^{6}$ \\
\hline
\end{tabular}




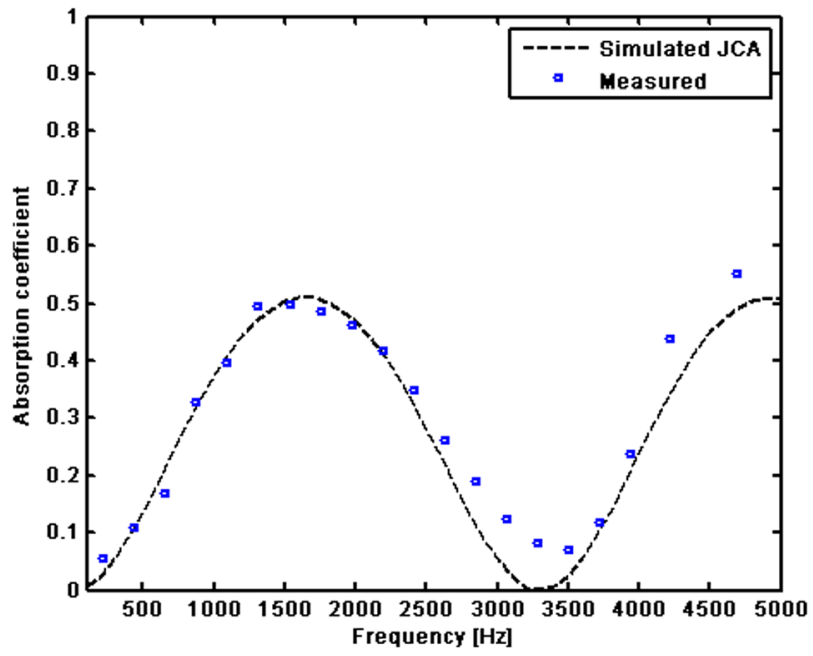

FIG. 8. (Color online) Normal incidence sound absorption coefficient of a mesh with parameters $\left(d_{2}, t_{2}, \phi_{2}, Y_{d}, D\right)=(36.38 \mu \mathrm{m}, 50 \mu \mathrm{m}, 27.23 \%, 33.34 \mu \mathrm{m}, 5 \mathrm{~cm})$.
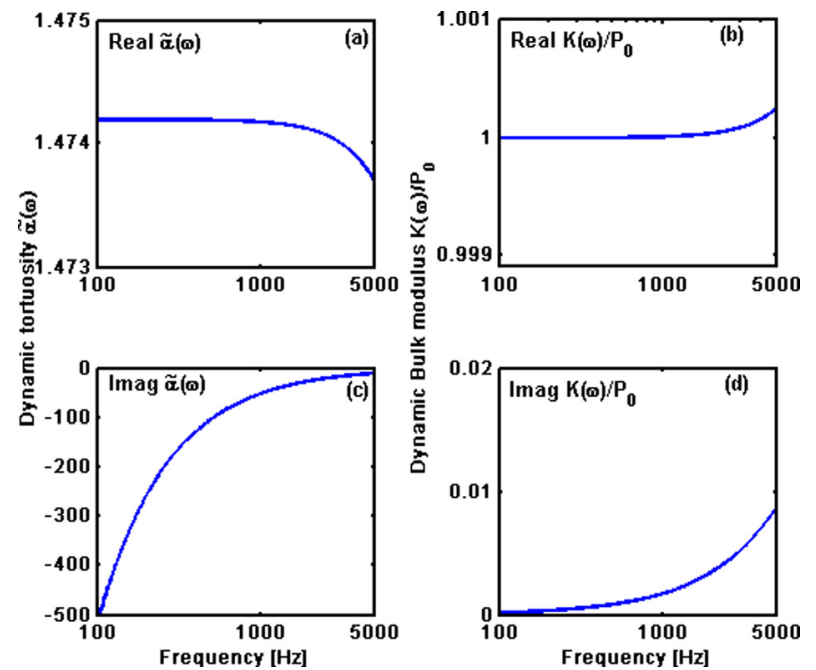

FIG. 9. (Color online) Dynamic tortuosity and bulk modulus of air for a precision woven micrometric mesh Sefar nitex 36/28. (a) and (b) real parts, (c) and (d) imaginary parts.

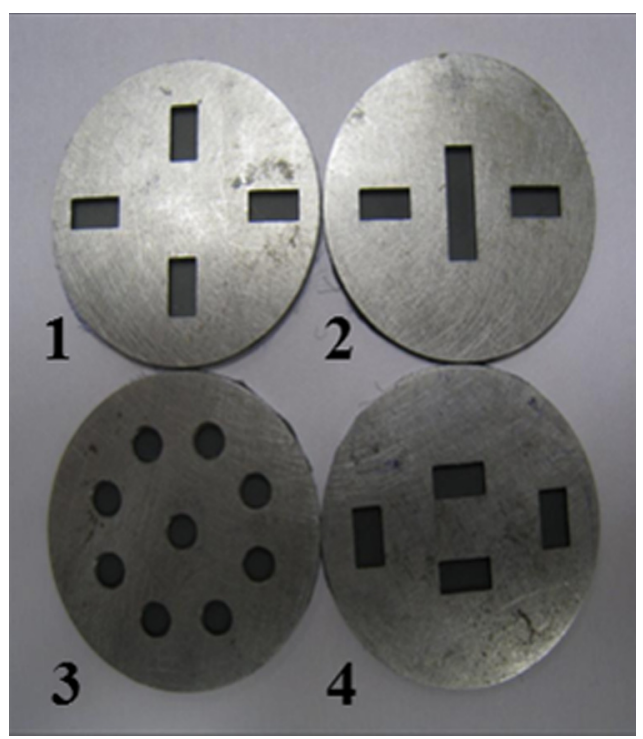

FIG. 10. (Color online) MPPs and MSPs with different perforations distribution.

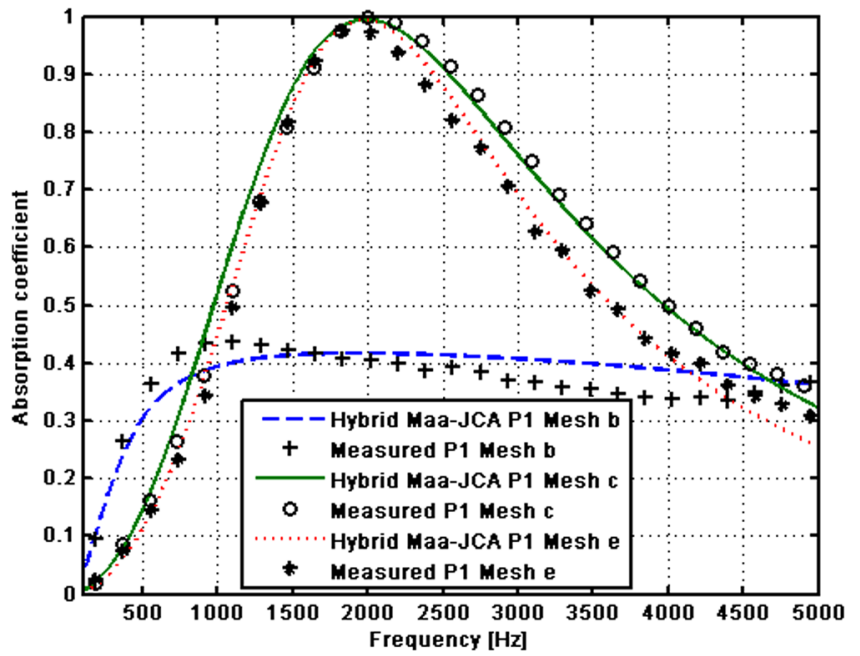

FIG. 11. (Color online) Normal incidence sound absorption coefficient of plate 1 combined with mesh $b(+), c(\circ)$, and $e(\times)$. Air cavity $D=2 \mathrm{~cm}$.

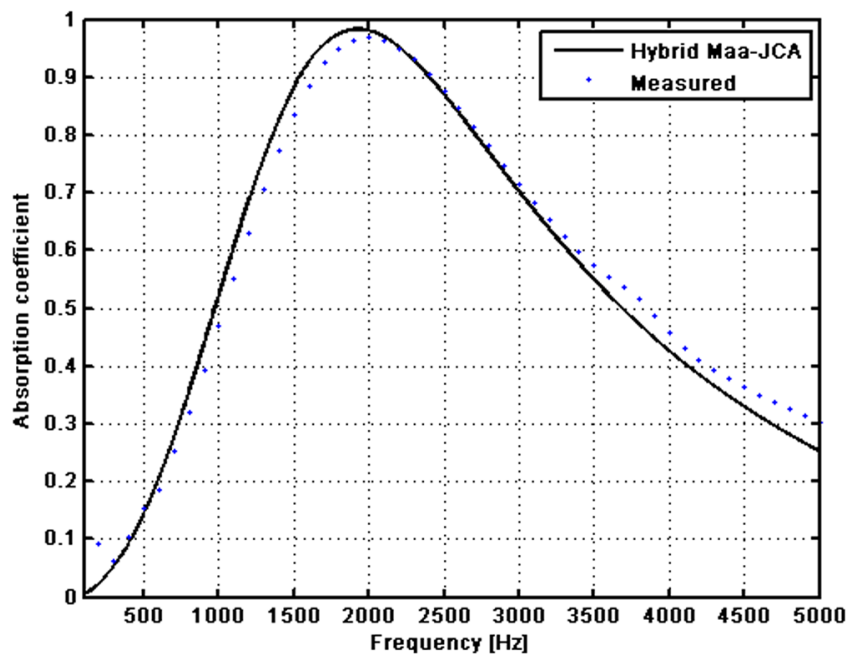

FIG. 12. (Color online) Normal incidence sound absorption coefficient of plate 3 combined with mesh $d$. Air cavity $D=2.4 \mathrm{~cm}$.

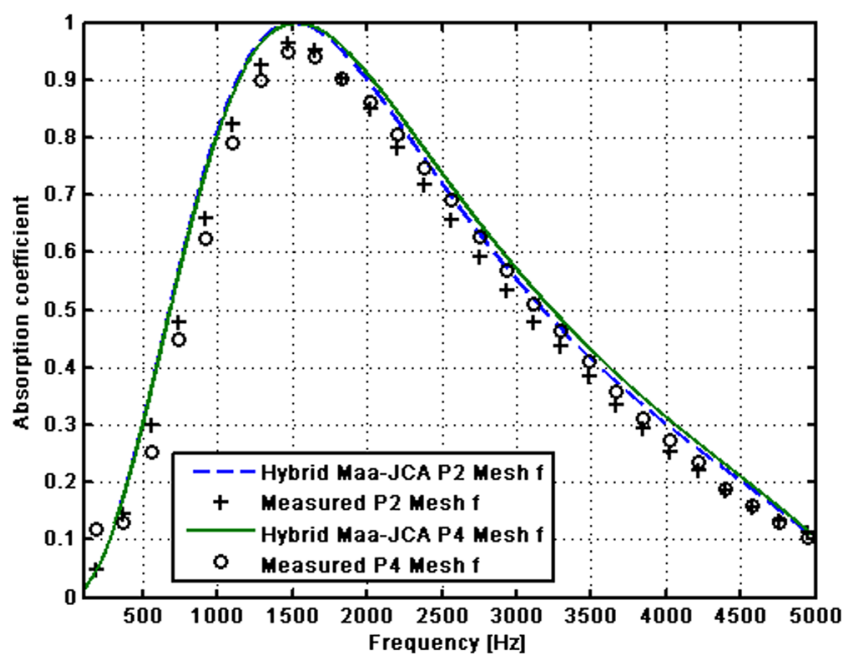

FIG. 13. (Color online) Normal incidence sound absorption coefficient of plate $2(+)$ and plate 4 (०) combined with mesh $f$. Air cavity $D=3.13 \mathrm{~cm}$.

Ruiz et al:: Perforated plates backed by woven meshes

3145 
between the two meshes is about ten times and the results are very similar. Figure 12 validates the model for a carrying plate with circular perforations and a woven mesh Sefar 41/31, mesh $d$. Mesh $f$ (Sefar HF 34-39) is then combined with plates 2 and 4 (P2-mesh $f$ and $\mathrm{P} 4$-mesh $f$; see Fig. 13). It appears that using the same mesh with two different plates with the same perforation ratio but different perforations distribution provides very similar absorption curve. Deviations from the two curves in Fig. 13 are due to the fact that the value of $\xi$ is smaller in plate 2 than in plate 4 , and therefore the attached mass in the former is slightly smaller than in the latter.

\section{CONCLUSIONS}

A hybrid acoustic model using a macroperforated carrying plate and a micrometric woven mesh has been proposed. The absorption curve of such a system depends on seven parameters: the constitutive parameters of the panel $\left(d_{1}, t_{1}, \phi_{1}\right)$, the thickness of the air cavity $(D)$, and the parameters of the micrometric mesh $\left(d_{2}, t_{2}, \phi_{2}\right)$.

A straightforward equation for the calculation of the thermal characteristic length with square pores was used. This equation resulted from a simple modeling of the woven mesh elementary cell. The result tends to show that the thermal effects have a great importance in woven meshes at high frequencies.

A correction of the air flow resistivity of the mesh when placed behind a carrying plate was proposed. This correction accounts for a "diaphragm effect" due to the squeezing of the air flow inside the macroperforation, which results in an effective flow resistivity for the mesh.

The transfer matrix method allows the handling of multilayer configurations. The macroperforated plate can be studied in the framework of the classical Maa model, while the porous layer can be modeled as an equivalent fluid with the JCA approach.

The costs of an absorbing system can be considerably reduced by using macroperforated panels and commercial meshes. Panels with perforations of the millimetric order are more manageable and can be drilled easily or even purchased. Micrometric meshes used for filtering are commercially manufactured and even woven curtains can be used for this purpose.

\section{ACKNOWLEDGMENTS}

This work has been supported by the Spanish Ministry of Science and Innovation (MICINN) through Project Nos. TRA2008-05654-C03-03 and TRA2011-26261-C04-01. The authors wish to thank Dr. D. Lafarge for stimulating discussions.

\section{APPENDIX}

A numerical calculation of the viscous characteristic length $\Lambda$ using the finite element method has been implemented under the assumptions of laminar, incompressible inviscid, and stationary flow. The fibers are modeled here as circular cylindrical rods fitted into each other [Fig. 2(a)]. For the boundary condition, a constant unit velocity field is imposed at the entrance.

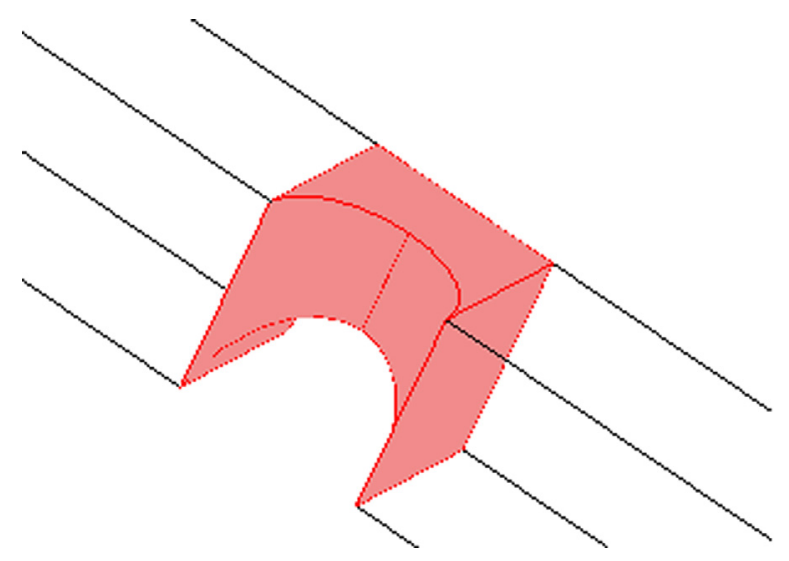

FIG. 14. (Color online) Air volume integration in 1/4 mesh

One-quarter of the modeled structure is shown in Figs. 14 and 15. Streamlines and flow vectors are represented (see Fig. 16). The velocity field is determined numerically and so the surface and volume speeds are calculated for the area in which the fluid passes through the woven mesh. The reference surface and volume are represented below (Figs. 15 and 14, respectively).

As an example of calculation for an entrance boundary unit speed equal to $\vartheta_{0}=1 \mathrm{~m} / \mathrm{s}$, the numerical calculation for Sefar nitex 36/28 (fiber diameter $Y_{d}=33.34 \mu \mathrm{m}$, step of the mesh $69.72 \mu \mathrm{m}$ ), yields $\Lambda / \Lambda^{\prime}=1.127$.

\section{Remarks}

This ratio $\Lambda / \Lambda^{\prime}$ greater than 1 is unexpected. It is due to the fact that the numerical integration of $\Lambda$ is performed in the elementary volume shown in the model of Fig. 2(b) using a constant unit velocity field at the entrance as boundary condition.

However, it is thought that this integration volume only corresponds to an approximate volume that should be used for the true material. Furthermore, the definition of $\Lambda$ implies that the calculation is averaged over a homogenization volume in which the proportion of air corresponds to the porosity while the real mesh is modeled as a single layer of intersecting cylinders, for which the definition of $\Lambda$ may be valid only approximately. This may explain why the results obtained for the ratio can be greater than 1 .

The values found for $\Lambda$ are close to half the interfiber distance (see Table I), which seems to confirm that the orders of magnitude are correct. These values are those used in the numerical simulation on the absorption coefficient.

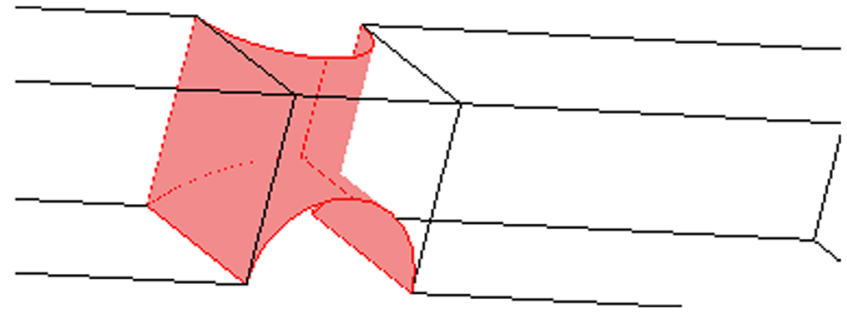

FIG. 15. (Color online) Surface of separation between the woven textile mesh and fluid (1/4 mesh). 


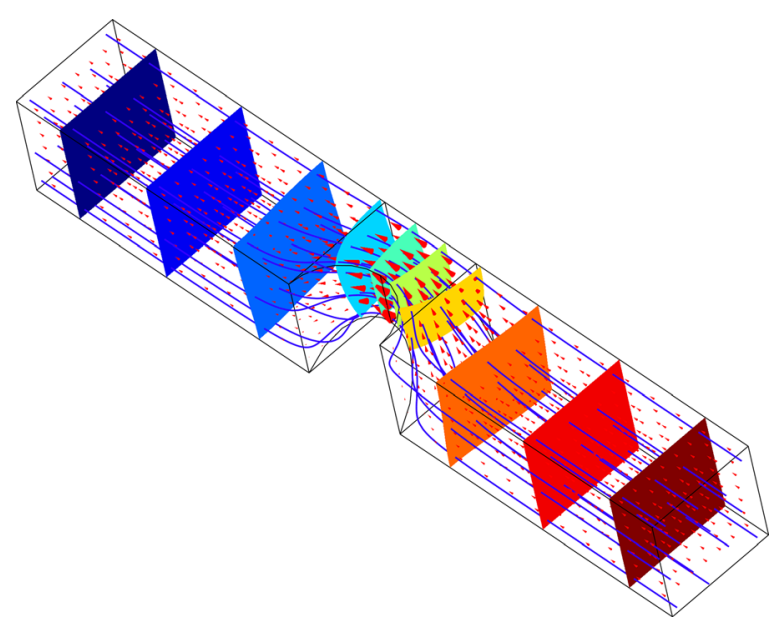

FIG. 16. (Color online) Speed field around and in the woven mesh. The increase in speed can be seen.

A confirmation of the validity of the numerical approach consists of the determination of the value of the ratio $\Lambda / \Lambda^{\prime}$ for a flow around a single cylinder. An analytical calculation in this configuration was proposed by Allard and Champoux in Ref. 24. The present numerical calculation using finite elements, under the same assumptions as in Ref. 24 , provides a ratio $\Lambda / \Lambda^{\prime}=0.503$, which is very close to the result of Allard and Champoux who found the ratio 0.5.

${ }^{1}$ D. Y. Maa, "Microperforated-panel wideband absorbers," Noise Control Eng. J. 29(3), 77-84 (1987).

${ }^{2}$ J. W. S. Rayleigh, The Theory of Sound (Dover, New York, 1945), pp. 1-319.

${ }^{3}$ J. B. Crandall, Theory of Vibrating Systems and Sound (Van Nostrand, New York, 1926), Appendix A.

${ }^{4} \mathrm{U}$. Ingard, "On the theory and design of acoustic resonators," J. Acoust. Soc. Am. 25(6), 1037-1061(1953).

${ }^{5}$ D. Y. Maa, "Theory of microslit absorbers," Acta Acust. 25(6), 481-485 (2000) (in Chinese).

${ }^{6}$ R. T. Randeberg, "Perforated panel absorbers with viscous energy dissipation enhanced by orifice design," Ph.D. thesis, Norwegian University of Science and Technology, Trondheim, Norway, 2000.
${ }^{7}$ J. F. Allard and N. Atalla, Propagation of Sound in Porous Media. Modelling Sound Absorbing Materials (Wiley, Chichester, UK, 2009), Chaps. 5, 9, 11.

${ }^{8}$ D. L. Johnson, J. Koplik, and R. Dashen, “Theory of dynamic permeability and tortuosity in fluid-saturated porous media," J. Fluid Mech. 176, 379402 (1987).

${ }^{9}$ Y. Champoux and J. F. Allard, "Dynamic tortuosity and bulk modulus in air-saturated porous media,” J. Appl. Phys. 70(4), 1975-1979 (1991).

${ }^{10}$ N. Atalla and J. F. Sgard, "Modeling of perforated plates and screens using rigid frame porous models," J. Sound Vib. 303, 195-208 (2007).

${ }^{11}$ J. Kang and H. V. Fuchs, "Predicting the absorption of open weave textiles and micro-perforated membranes backed by an air space," J. Sound Vib. 220(5), 905-920 (1999).

${ }^{12}$ J. Pfretzschner, P. Cobo, F. Simon, M. Cuesta, and A. Fernández, "Microperforated insertion units: An alternative strategy to design microperforated panels," Appl. Acoust. 67, 62-73 (2006).

${ }^{13} \mathrm{~F}$. Chevillote, "Controlling sound absorption by an upstream resistive layer," Appl. Acoust. 73, 56-60 (2012).

${ }^{14}$ P. Cobo, M. Cuesta, and M. Siguero, "Comparison of models describing double layer microperforated absorbers," Noise Control Eng. J. 57, 10-15 (2009).

${ }^{15}$ M. R. Stinson and Y. Champoux, "Propagation of sound and the assignment of shape factors in model porous materials having simple pore geometries," J. Acoust. Soc. Am. 91(2), 685-695 (1992).

${ }^{16}$ T. H. Melling, "The acoustic impedance of perforates at medium and high sound pressure levels," J. Sound Vib. 29(1), 1-65 (1973).

${ }^{17}$ R. Tayong, T. Dupont, and P. Leclaire, "Experimental investigation of holes interaction effect on the sound absorption coefficient of microperforated panels under high and medium sound levels," Appl. Acoust. 72, 777-784 (2011).

${ }^{18}$ D. Y. Maa, "Theory and design of microperforated panel sound-absorbing constructions," Sci. Sin. 18, 55-71 (1975).

${ }^{19}$ H. Ruiz and P. Cobo, "Proposal of alternative designs for microperforated panels," in Proceedings of the 40th International Congress and Exposition on Noise Control Engineering, Internoise. Osaka, Japan (September 4-7, 2011).

${ }^{20}$ L. Jaouen and F.-X. Bécot, "Acoustical characterization of perforated facings," J. Acoust. Soc. Am. 129(3), 1400-1406 (2011).

${ }^{21}$ J. P. Dalmont, C. J. Neverveen, and N. Joly, "Radiation of tubes with different flanges: Numerical and experimental investigations," J. Sound Vib. 244(3), 505-534 (2001).

${ }^{22}$ D. H. Lee and P. Kwon, "Estimation of the absorption performance of multiple layer perforated panel systems by transfer matrix method," J. Sound Vib. 278, 847-860 (2004).

${ }^{23}$ J. Y. Chung and D. A. Blaser, "Transfer function method of measuring in-duct acoustic properties. I. Theory," J. Acoust. Soc. Am. 68(3), 907913 (1980).

${ }^{24}$ J. F. Allard and Y. Champoux, "New empirical equations for sound propagation in rigid frame fibrous materials," J. Acoust. Soc. Am. 91(6), 3346-3353 (1992). 